\title{
I 00 Prognostic significance of post-procedural irreversible myocardial injury detected by cardiovascular magnetic resonance imaging
} Joseph B Selvanayagam*1, Kazem Rahimi², Adrian Banning3, Adrian SH Cheng ${ }^{2}$, Tammy J Pegg ${ }^{2}$, Theodoros Karamitsos ${ }^{2}$, David P Taggart ${ }^{2}$ and Stefan Neubauer ${ }^{2}$

Address: ${ }^{1}$ Flinders University, Adelaide, Australia, ${ }^{2}$ Oxford University, Oxford, UK and ${ }^{3}$ John Radcliffe Hospital, Oxford, UK

* Corresponding author

from I I th Annual SCMR Scientific Sessions

Los Angeles, CA, USA. I-3 February 2008

Published: 22 October 2008

Journal of Cardiovascular Magnetic Resonance 2008, I0(Suppl I):AI doi:I0.II86/I532-429X-I0-SI-AI

This abstract is available from: http://jcmr-online.com/content/I0/SI/AI

(c) 2008 Selvanayagam et al; licensee BioMed Central Ltd.

\section{Introduction}

Controversy remains over the prognostic significance of revascularization procedure related myocardial injury. Delayed enhancement cardiovascular magnetic resonance imaging (DE-CMR) has been shown to reliably identify areas of irreversible myocardial injury.

\section{Purpose}

In the present study, we evaluated the prognostic significance of procedure related myocardial injury in a consecutive series of patients undergoing high risk PCI or CABG.

\section{Methods and Results}

152 patients underwent DE-CMR for assessment of new irreversible injury pre and 1 to 6 days post elective PCI or CABG. Primary endpoint was defined as total mortality, non-fatal myocardial infarction (MI), ventricular arrhythmia terminated by ICD (VA), and unstable angina or heart failure requiring hospitalization. Secondary endpoint was the composite of total mortality, non-fatal MI and VA. During a median follow-up of 2.9 years, 27 patients $(18 \%)$ reached the primary endpoint and 12 patients $(8 \%)$ the secondary endpoint. 49 patients $(32 \%)$ had evidence of new myocardial hyperenhancement (HE) with a median mass of $5.0 \mathrm{~g}$ (IQR 2.7-9.8). In a univariate analysis, age, LV ejection fraction post intervention, and presence of new HE were predictive of the primary outcome.
Elevated troponin (at $24 \mathrm{~h}$ ) showed a trend towards poorer outcome. In a multivariate Cox regression analysis only age and presence of new HE (Hazard ratio 2.7, 95\% CI 1.03, 5.79) remained independently correlated with occurrence of the primary endpoint. New myocardial HE was the single independent predictor of the composite secondary endpoint (HR 4.2, 95\% CI 1.07, 16.12).

\section{Conclusion}

Even small amounts of procedure-related myocardial injury are associated with poorer medium term clinical outcomes. CMR identified myocardial injury may be a stronger prognostic marker than cardiac troponin in the setting of coronary revascularization. 